\title{
siRNA-dependent Gene Silencing of EVI1 Decreased Cell Proliferation in MKN45 Cells
}

\author{
Yuri Na ${ }^{1}$, Hyejun Hwang ${ }^{2}$, Jaehyuk $\mathrm{Cho}^{3}$, Woo Rin Lee ${ }^{4, *}$ \\ ${ }^{1}$ Albany Academy for Girls, New York, The United States of America \\ ${ }^{2}$ North London Collegiate School Jeju, Jeju, Republic of Korea \\ ${ }^{3}$ Holy Cross High School, New York, The United States of America \\ ${ }^{4}$ Department Biological Science, University of Suwon, Hwaseong, Republic of Korea
}

Email address:

nayuri0405@gmail.com (Y. Na),jisoohwang04@gmail.com (H. Hwang),jaecho0913@gmail.com (J. Cho), gosyber1@gmail.com (W. R. Lee)

${ }^{*}$ Corresponding author

\section{To cite this article:}

Yuri Na, Hyejun Hwang, Jaehyuk Cho, Woo Rin Lee. siRNA-dependent Gene Silencing of EVI1 Decreased Cell Proliferation in MKN45 Cells. Cell Biology. Vol. 8, No. 2, 2020, pp. 27-32. doi: 10.11648/j.cb.20200802.12

Received: September 14, 2020; Accepted: September 29, 2020; Published: October 16, 2020

\begin{abstract}
MDS1 and EVI1 complex locus protein EVI1 (MECOM) is an oncogenic transcription factor in many types of cancer. However, the clinical significance of MECOM in esophagogastric cancer has not been well elucidated. The aim of this study was to define the association of amplification of MECOM and esophagogastric cancer patients' overall survival rate and to investigate the functional role of MECOM in MKN45 cell proliferation and apoptosis. In this study, a total of 3236 esophagus and gastric patients were analyzed from 16 genomic studies using cBioPortal, which provides an open-access resource with multiple cancer genomics data sets. When the patient samples were divided into two subgroups (MECOM amplified group and $M E C O M$ non-amplified group), the poor survival rate was significantly associated with MECOM amplification $(p=0.04)$. To investigate the role of EVI1 on esophagogastric cancer cells, siRNA dependent gene silencing of EVI1 in MKN45 cells was conducted. RT-PCR was used to examine the expression of EVI1 in MKN45 cells. The MTT assay was used to examine cell proliferation. The apoptotic cells were quantified by fluorescence cell counter. Knockdown of EVI1 leads to reduced cell proliferation and induced apoptosis in MKN45 cells. These results indicate that MECOM may be a potential target for esophagus and gastric cancer gene-targeted therapy. Collectively, our result suggested that EVI1 is a probable target gene for esophagogastric cancer cell proliferation.
\end{abstract}

Keywords: MECOM, Esophagogastric Cancer, siRNA, RT-PCR, Cell Proliferation, Apoptosis

\section{Introduction}

The esophagus and stomach are the parts of the upper gastrointestinal (GI) tract and both show similar features [1]. Esophagogastric cancer shows exceptionally high rates of death limited to Asia. Gastric cancer in the early stage is typically small and shows less symptoms than other cancers which can delay the diagnosis. Therefore, the mortality rate of gastric cancer increases rapidly, leading it to be the second deadliest cancer worldwide [2]. Meanwhile, esophageal cancer displays a dangerous presentation which includes unresectable carcinoma or metastases, which is visible through radiographic technology [3]. Moreover, the detection and prevention of the cancer is very complicated since it shows two histologic types: squamous cell carcinoma (SCC) and adenocarcinoma (ACE) [4].

$M E C O M$ (MDS1 and EVI1 complex locus) is a gene that codes for an oncoprotein or serves as a transcriptional regulator [5]. The gene encodes for many different isoforms, including transcription factor variants such as EVI1, EVI1 $\Delta 324$, and MDS1-EVI1 [5].

The main oncogenic transcription factor, EVI1, has two DNA-binding Zinc Finger (ZNF) domains: a domain in the Nterminus to a GATA-like DNA sequence and a domain in the Cterminus to an E-twenty-six family (ETS) like motif [6]. Previous studies indicate that the N-terminal ZNF domain of EVI1 is binding to sites of frequent chromosomal recombination 
rates, which explains EVI1's potential for genomic instability and oncogenic characteristics [6]. According to previous studies, however, some EVI1's binding sites were discovered to be far from the actual gene, which raised questions on its transcription regulating function [6]. EVI1 is known to be involved in acute myeloid leukemia (AML) and various types of cancers like ovarian carcinoma with a low chance of survival [7].

Unlike EVI1 which has two DNA ZNF-binding domains, EVI1 $\Delta 324$ protein has only one [5]. The EVI1 $\Delta 324$ protein has a disrupted N-terminal ZNF domain. It normally binds to the ETS-like DNA binding site, CCATCTCC, with its Cterminus ZNF domain [5]. Still, according to previous studies, the transcriptional activity of EVI1 and EVI1 $\Delta 324$ variants were very similar in HeLa cells, indicating minimal impact of EVI1 N-terminus ZNF domain to transcriptional regulation [5]. Both variants also had similar effects on gene expression related to the identical C-terminal ZNF domain used to bind to DNA [5]. EVI1 $\Delta 324$ expression also showed increased anchorage-independent growth in HeLa cells: an oncogenic property like EVI1 [5].

Interestingly, while the two variants, EVI1 and EVI1 $\Delta 324$, show oncogenic characteristics and low patient survival, the MDS1-EVI1 variant, a longer form of EVI1 containing the MDS1 gene with an extended 188 amino acid N-terminus, shows tumor suppressing properties $[5,7,8]$. In an earlier study with hematopoietic and progenitor (stem) cells, the MDS1-EVI1 was found to repress cell growth whereas EVI1$145 \mathrm{kDa}$ isoform (of EVI1 isoform) maintained and prolonged cell growth [6]. In another study of bone marrow of leukemia patients, the rearrangement of chromosomes (3p26) showed to be crucial in the level of isoform expressions [8]. While there was low EVI1 and MDS1-EVI1 expression in normal bone marrows, there was very high EVI1 expression and the normal low MDS1-EVI1 expression after the chromosomal rearrangement [8]. These two examples illustrate both oncogenic and tumor-suppressing capabilities of MECOM and suggest its impact on cell proliferation.

MDS1-EVI1 demonstrated antagonistic characteristics with the EVI1 gene: activation of MDS1-EVI1 was repressed by the expression of the EVI1 [8]. It was also found that $M E C O M$ itself strictly regulates its own expression: EVI1$145 \mathrm{kDa}$ isoform activated transcription whereas EVI1 $\Delta 324$ and MDS1-EVI1 repressed transcription [6].

The various isoforms of MECOM have been studied in many different cancers, but not specifically in esophagogastric cancer. The effect of amplification of $M E C O M$ genes on the esophagogastric cancer cell has not been studied. The purpose of this study is to determine the functional role of $M E C O M$, especially EVI1, on esophagogastric cancer cells which may affect esophagogastric cancer patients' survival rate.

\section{Materials and Methods}

\subsection{Data Source and Patient Selection}

The cBioPortal for cancer genomics is an open-access resource for multidimensional cancer genomics data sets. (http://www.cbioportal.org). It provides visualization and analysis of large-scale cancer genomics data sets from multiple studies [9]. This database provides visualization and analyzing tools for more than 6,000 tumor samples from 290 cancer studies. 16 genomic studies of esophagogastric cancer were found in the cBioPortal for cancer genomics.

\subsection{Patient Survival Analysis of MECOM}

Amplification as well as survival data and clinical profiles were analyzed from the cBioPortal website after submitting the query "MECOM: AMP" in the input box. Altogether, 3236 esophagus and gastric patients were analyzed. The selected cohort was further analyzed by two groups: $M E C O M$ amplified group vs $M E C O M$ non-amplified group (amplification indicates a high-level amplification of gene). Overall survival (OS) was calculated on the basis of cBioPortal's online instruction. No statements of approval or informed consent were required for the study as it obtained data from an open access database.

\subsection{Cell Line and Culture}

Human gastric cancer cell line MKN45 was obtained from the Korean Cell Line Bank. MKN45 cells were maintained in Gibco RPMI-1640 medium (Life Technologies) supplemented with $10 \%$ HyClone fetal bovine serum (Thermo Fisher) and $1 \%$ penicillin and streptomycin in a 5\% $\mathrm{CO}_{2}$ atmosphere at $37^{\circ} \mathrm{C}$ incubator.

\section{4. $R N A$ Interference}

Predesigned siRNA (2122-1) were purchased from Bioneer to deplete EVI1 expression. The negative control scrambled siRNA med GC (12935-300) was purchased from Invitrogen. Cells were transfected with $20 \mathrm{nM}$ siRNAs using lipofectamine RNAimax reagent (Invitrogen) with 1:3 ratio of siRNA $(\mu \mathrm{g})$ to lipofectamine $(\mu \mathrm{l})$.

\subsection{Total RNA Isolation and Reverse Transcription}

Total cellular RNA was extracted with Total RNA extraction spin kit (Intron) and treated with DNase I (Invitrogen). The cDNA was reverse transcribed from $1 \mu \mathrm{g}$ of total RNA using oligo (dT) primers according to the manufacturer's protocol (Enzynomics).

\subsection{Polymerase Chain Reaction}

Polymerase chain reaction was performed in $20 \mu \mathrm{l}$ reaction with PCR-Premix (Bioneer). The PCR mixture was prepared according to the manufacturer's protocol. The primers from the previous study were used for the amplification of cDNA from each of two human genes [10]. PCR was performed with cycles of $94^{\circ} \mathrm{C}$ for $15 \mathrm{~s}, 55^{\circ} \mathrm{C}$ to $60^{\circ} \mathrm{C}$ for $15 \mathrm{~s}$, and $74^{\circ} \mathrm{C}$ for 30 s. The forward primer 5'GGTCAACAAACCAATTTAGACAGACA - $3^{\prime}$ and reverse primer 5'- TTCAGAATGAGGCGACGATGT - 3 ' yielded a 87-base pair (bp) product from EVI1 using an annealing 
temperature $52^{\circ} \mathrm{C}$. For GAPDH, a (187) bp product was produced by forward primer 5'TGGAGAAGGCTGGGGCTCAT -3' and reverse primer 5'GACCTTGGCCAGGGGTGCTA -3'.

\subsection{Gel Electrophoresis and Data Acquisition}

The $1.2 \%$ agarose gels were pre-stained with RedSafe nucleic acid staining reagent (Intron). Aliquots taken from PCR reactions were electrophoresed on agarose gels at 100 volts for $25 \mathrm{~min}$. The gel electrophoresis images were captured with a digital camera, and the densitometry feature of Image studio version 5.2 was used to measure band intensities. The relative intensities of EVI1 bands were normalized by the GAPDH band.

\subsection{Cell Proliferation Assay (MTT Assay)}

The 3-(4,5-dimethylthiazol-2-yl)-2,5-diphenyltetrazolium bromide (MTT) assay was performed using PrestoBlue Cell Viability Reagent (Invitrogen). Ten thousand cells were grown in a 96 well plate for $72 \mathrm{~h}$. MTT solution was added to the culture medium, and cells were incubated at $37^{\circ} \mathrm{C}$ for 30 min. The absorbance was measured at $570 \mathrm{~nm}$ using Fluoroskan $^{\mathrm{TM}}$ Ascent microplate reader (Thermo Fisher). A blank reference was measured from the control wells without cells. Data were obtained from three independent experiments in triplicate.

\subsection{Fluorescent Cell Count}

The cells were trypsinized after siRNA transfection and the percentage of apoptotic cells were measured by counting cells, which were stained by acridine orange/propidium iodide (Logos Biosystems), an apoptosis indicator, with the Luna FL Dual Fluorescence Cell Counter (Logos
Biosystems)

\subsection{Statistical Analysis}

All statistical analysis was performed using SPSS 13.0 (SPSS) and Excel (Microsoft). Quantitative data were expressed as mean \pm SD (standard deviation). Survival curves were plotted using the cBioPortal. All results are displayed with $P$ values from a log-rank test. For gene expression and cell proliferation analysis, Student's $t$-test was used to compare two samples. $P$ values of $<0.05$ were considered to be statistically significant.

\section{Result}

\subsection{Amplification of MECOM Decreases the Overall Survival of Brain Cancer Patients}

Gene amplification indicates the expansion in the copy number of a gene within the genome of a cell [11]. It is frequently associated with aggressive behavior of the tumor and poor prognosis [11]. According to the public clinical data from cBioPortal, MECOM was frequently amplified in $9.85 \%(221 / 2244)$ of patients with esophagogastric cancer (Figure 1). When patients were divided into two groups (MECOM amplified group and MECOM non-amplified group), the amplified group showed a marginally significant association with decreased overall survival $(\mathrm{p}=0.04)$, suggesting that the amplified $M E C O M$ may be a risk factor of esophagogastric cancer (Figure 1). The median survival month of amplified MECOM showed 22.8 months compared to 31.3 months in the non-amplified $M E C O M$ group (Figure 1). This result implied that $M E C O M$ amplification may positively correlate with esophagogastric cancer progression.

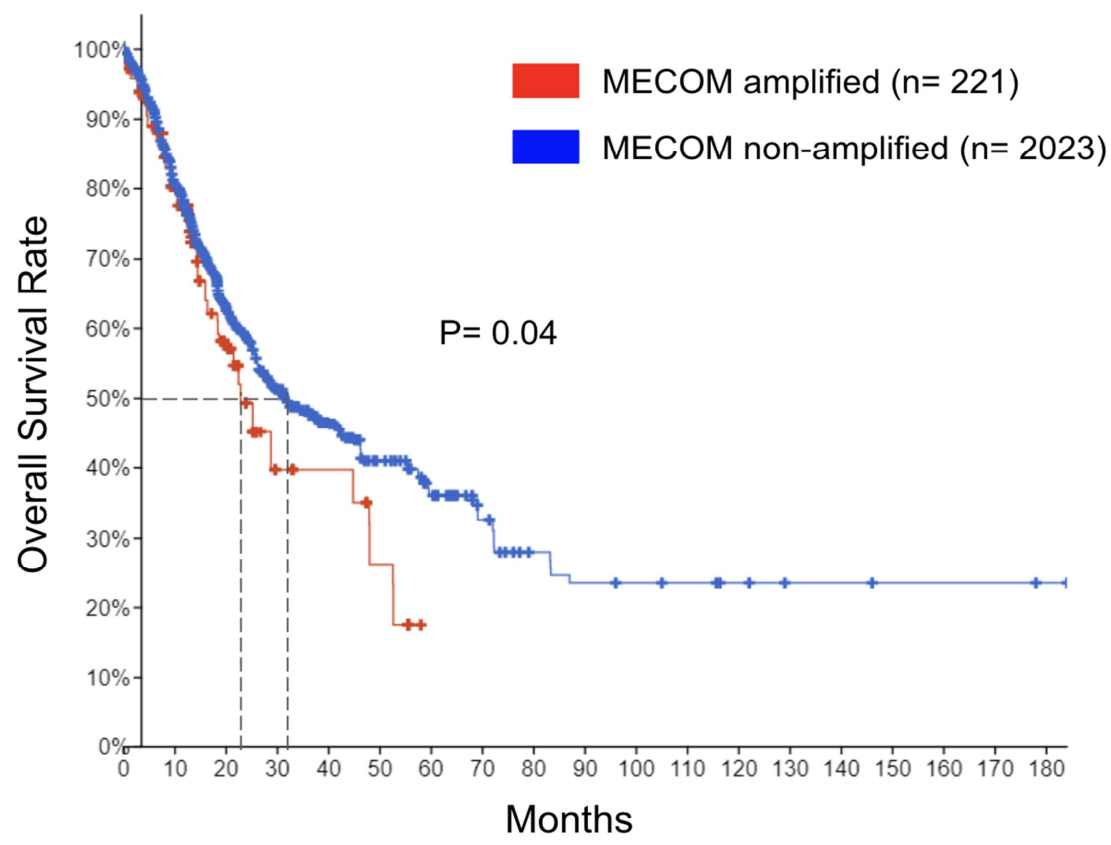

Figure 1. MECOM amplification enhances patient survival rate. The MECOM amplified group (red) and wild type group (blue) were 221 and 2023 patients respectively. Out of the total patients, the amplified group lived a median 22.8 months overall. The wild type group lived a median 31.3 months. 


\section{2. siRNA Targeting EVI1 Decreased mRNA Expression Level of EVI1 and Inhibits Cell Proliferation}

Amplification leads to the increased expression of genes, which may provide a selective advantage for cancer progression [12]. Previous study showed that only the expression level of EVI1, but not the fusion transcript MDS1-EVI1 or MDS1, was overexpressed in hepatocellular carcinoma [13]. EVI1 antagonizes transforming growth factor- $\beta$-mediated growth inhibition [13]. To understand the functional role of EVI1 in cancer, MKN45 gastric cancer cells were transfected with small interfering RNA targeting EVI1 (siEVI1). One siRNA for negative control (siControl), which is designed not to target any gene, and siEVI1 were used in this experiment to validate the effect of siRNA on EVI1 mRNA expression level. The effect of siRNA transfections on EVI1 expression was confirmed using quantitative polymerase chain reaction (qPCR) (Figure 2A). The result indicates that siRNA targeting EVI1 decreased mRNA expression level of EVI1 in MKN45 cells (Figure 2B). To find the functional role of EVI1 in MKN45 cells, cell proliferation was analyzed by MTT assay. EVI1 depletion significantly inhibited cell proliferation at $72 \mathrm{~h}$ after transfection (Figure 2C). This result was consistent with the previous studies that suggested EVI1 promoted cell proliferation in ovarian cancer, prostate cancer, and pancreatic cancer [14-16].
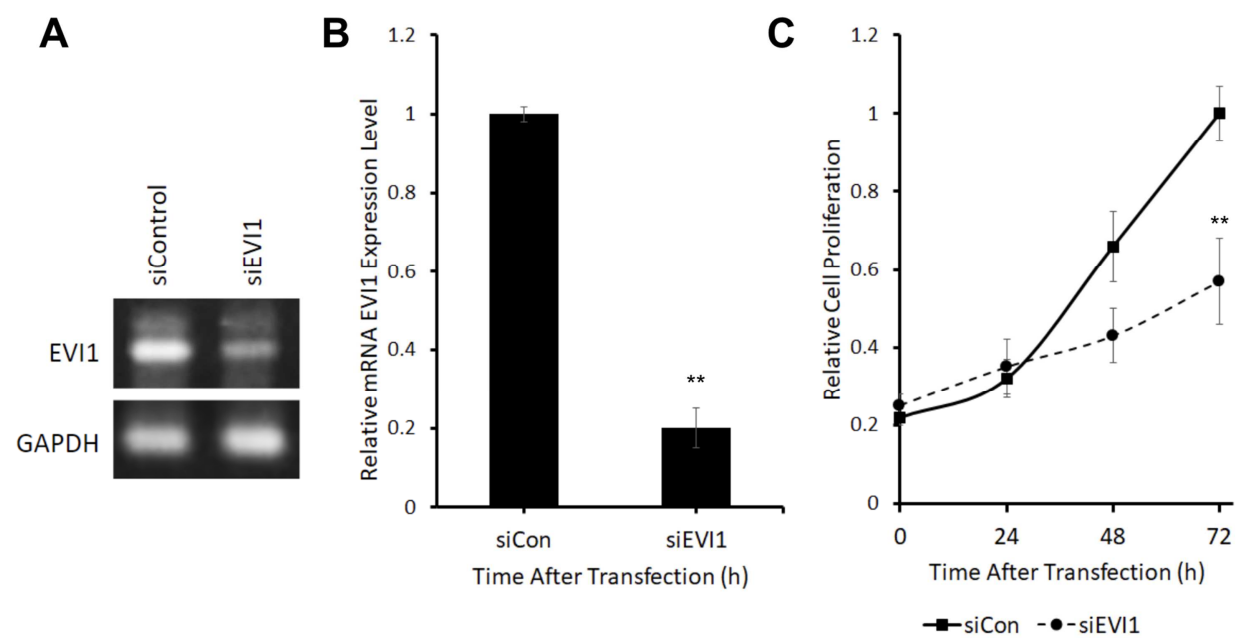

Figure 2. siRNA targeting EVII decreased the mRNA of EVII and inhibited cell proliferation on MKN45 cells. (A) qPCR analysis showing the mRNA levels of siControl (negative control) and siEVII transfected MKN45 cells at $48 \mathrm{~h}$ after transfection. (B) qPCR analysis of EVII mRNA levels at $48 \mathrm{~h}$ after transfection (C) Cell proliferation analysis by MTT assay $(n=3$, mean $\pm S D)$. Student's $t$ test, ${ }^{* *}<p 0.01$.

\subsection{EVI1 Depletion Promoted Apoptosis in MKN45 Cells}

Cell proliferation is determined by the difference between cell replication and cell death [17]. To find out whether the inhibitory effect of proliferation was mediated by cell death, apoptosis, programmed cell death, was analyzed in siEVI1 transfected MKN45 cells. The immunofluorescence image was captured at $72 \mathrm{~h}$ after transfection of siControl and siEVI1 (Figure 3A). The siControl transfected cells showed normal structure without prominent apoptosis with green fluorescence, which indicates live and healthy cells (Figure $3 \mathrm{~A})$. The cells generating red fluorescence cells, which is stained by propidium iodide, were observed in siEVI1 transfected cells (Figure 3A). When apoptotic cells were quantified by fluorescence cell counters, apoptotic cells were significantly increased in siEVI1 transfected cells (Figure 3B). Since EVI1 depletion promoted cell apoptosis of MKN45 cells, this result indicates that EVI1 elicits its inhibitory effect on cell proliferation by inducing apoptosis.
A

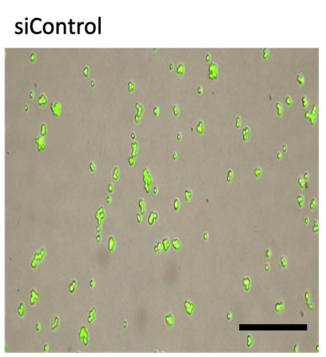

SiEVI1

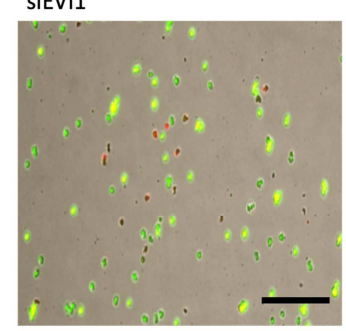

B

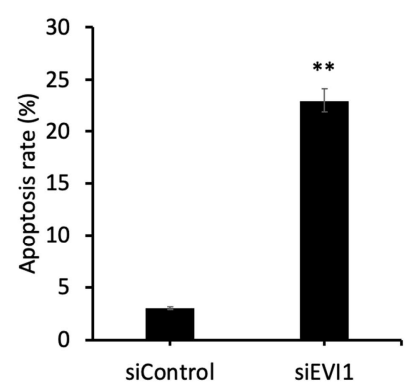

Figure 3. EVII depletion induced apoptosis of MKN45 cells. (A) Immunofluorescence image after acridine orange and propidium iodide double-staining. The cell image was analyzed at $72 \mathrm{~h}$ after siRNA transfection. Live cells generate green fluorescence and apoptotic cells generate red fluorescence. Scale bar, 100 $\mu m$. Magnification, x100. (B) Quantification of apoptotic cells. Quantitative data are presented $(n=3$, mean $\pm S D)$. Student's t test, $* *<p 0.01$. 


\section{Discussion}

The previous study identified that the MECOM amplification had been observed at myeloid malignancies and that MECOM amplification associated with MECOM mRNA overexpression [18]. Through the experiment of the study, all patients with MECOM amplification showed $M E C O M$ mRNA overexpression with a range of 2.6-54.4, whereas the median $M E C O M$ mRNA expression in patients without $M E C O M$ rearrangement/amplification was of a range 0.01-0.58 [18]. This indicates that there is a significant correlation between MECOM amplification and the MECOM mRNA overexpression [18]. In addition, amplified MECOM is associated with ovarian cancer [19]. MECOM activates PI3K and PKC, elevates mRNA and protein levels, when amplified at its location [19]. Its protein level is closely parallel to transcript levels and DNA copy numbers, when overexpressed MECOM promotes ovarian cell proliferation [19]. In this study, we found that MECOM amplification was associated with decreased patient survival rate. Therefore, $M E C O M$ amplification in esophagogastric cancer patient's tumors may have a high MECOM expression level, which leads to tumor progression.

Next, we found that EVI1 depletion inhibited cell proliferation by inducing apoptosis in MKN45 cells. This result is consistent with various cancer studies that showed EVI1 promoted cell growth by faster advancement through cell cycle and suppression of apoptosis [20, 21]. In an experiment with EVI1 in hematopoietic cells in mice, EVI1 overexpression was accompanied by increased cdk2 activity, heightened levels of hyperphosphorylated $\mathrm{Rb}$ protein, decreased p27 protein levels, and shortened G1 phase [22]. Re-expression of EVI1 could cause normal proliferation of HSCs. GATA-2 mRNA expression, crucial for cell proliferation, was also found reduced in EVI1-absent in HSCs [22]. For a study with lymphoblastic leukemia cells, EVI1 was found to regulate expression of apoptosis-related genes like XIAP or BCL2 [23]. When EVI1 gene expression was repressed, apoptosis sensitivity of the leukemia cells was increased and their proliferation tendencies were weakened [23]. In Hilar Cholangiocarcinoma (HCCA), EVI1 repression reduced cell proliferation, promoted apoptosis, and blocked cell cycle progression. EVI1 could also regulate PTEN levels in the AKT signaling pathway of HCCA [20]. Since the genes related to cell cycle and apoptosis were not investigated in this paper, the detailed molecular mechanism of EVI1 with cell cycle and apoptosis regulating genes should be further analyzed in the future.

Not only does the MECOM promote cell proliferation, it also promotes metastasis. PBK, a PDZ-binding kinase enabling cancerous cells to grow, targets EVI1 and promotes metastasis and resistance to ovarian cancer cure [19]. EVI1 is associated with triple-negative breast cancer, which is a cancer that tests negative for estrogen receptors, but enhances metastasis rate and apoptosis resistance by inducing anti- apoptotic genes. Similarly, in prostate cancer, EVI1 expression has impacts on proliferation and distance metastasis [24]. In nasopharyngeal carcinoma (NPC) tissues, EVI1 expression was positively correlated with poor prognoses and even caused chemo- or radio-resistance in NPC cells [25]. EVI1 also represses tumor-suppressing (Ecadherin) expression by forming a corepressor with snail and HDAC1, eventually reinforcing epithelial mesenchymal transition (EMT) of NPC cells [25]. Since there are many researches indicating that $M E C O M$ is associated with metastasis, the role of $M E C O M$ in esophagogastric cancer metastasis should be investigated further.

\section{Conclusion}

This study has several acknowledged limitations: further experiments to identify the genetic and epigenetic regulatory mechanisms of the $M E C O M$ are needed. The associations between the MECOM gene and other MECOM isoforms or genetic mechanisms were not validated, and the protein expression level was not analyzed. Another limitation is that the effect of EVI1 depletion on MKN45 cells should be validated by different esophagogastric cancer cell lines to evaluate both its prognostic significance. Despite the limitations, we were able to demonstrate the prognostic potential of the MECOM by analyzing public data provided by cBioPortal. When EVI1 was depleted by siRNA, it inhibited cancer cell proliferation by inducing apoptosis. Since MECOM functions as an oncogene, amplification or increased expression of $M E C O M$ may decrease the survival rates of esophagogastric cancer patients.

\section{References}

[1] Khushalani N. Cancer of the esophagus and stomach. Mayo Clin Proc. 2008; 83: 712-722.

[2] Leung WK, Wu M, Kakugawa Y, Kim JJ, Yeoh K, Goh KL, et al. Screening for gastric cancer in Asia: current evidence and practice. Lancet Oncol. 2008; 9: 279-287. doi: 10.1016/S1470-2045(08)70072-X.

[3] Enzinger PC, Mayer RJ. Esophageal cancer. N Engl J Med. 2003; 349: 2241-2252.

[4] Holmes RS, Vaughan TL. Epidemiology and pathogenesis of esophageal cancer. Semin Radiat Oncol. 2007; 17: 2-9.

[5] Sayadi A, Jeyakani J, Seet SH, Wei CL, Bourque G, Bard FA, et al. Functional features of EVI1 and EVI1 $\Delta 324$ isoforms of MECOM gene in genome-wide transcription regulation and oncogenicity. Oncogene. 2016; 35: 2311-2321.

[6] Maicas M, Vázquez I, Alis R, Marcotegui N, Urquiza L, Cortés-Lavaud X, et al. The MDS and EVI1 complex locus (MECOM) isoforms regulate their own transcription and have different roles in the transformation of hematopoietic stem and progenitor cells. Biochim Biophys Acta Gene Regul Mech. 2017; 1860: 721-729. 
[7] Nanjundan M, Nakayama Y, Cheng KW, Lahad J, Liu J, Lu K, et al. Amplification of MDS1/EVI1 and EVI1, located in the 3q26.2 amplicon, is associated with favorable patient prognosis in ovarian cancer. Cancer Res. 2007; 67: 30743084 .

[8] Soderholm J, Kobayashi H, Mathieu C, Rowley JD, Nucifora G. The leukemia-associated gene MDS1/EVI1 is a new type of GATA-binding transactivator. Leukemia. 1997; 11: 352358.

[9] Gao J, Aksoy BA, Dogrusoz U, Dresdner G, Gross B, Sumer $\mathrm{SO}$, et al. Integrative analysis of complex cancer genomics and clinical profiles using the cBioPortal. Sci Signal. 2013; 6: pl1.

[10] Luesink M, Hollink IHIM, van der Velden VHJ, Knops RHJN, Boezeman JBM, de Haas V, et al. High GATA2 expression is a poor prognostic marker in pediatric acute myeloid leukemia. Blood. 2012; 120: 2064-2075.

[11] Matsui A, Ihara T, Suda H, Mikami H, Semba K. Gene amplification: mechanisms and involvement in cancer. Biomol Concepts. 2013; 4: 567-582.

[12] Hyman E, Kauraniemi P, Hautaniemi S, Wolf M, Mousses S, Rozenblum E, et al. Impact of DNA amplification on gene expression patterns in breast cancer. Cancer Res. 2002; 62: $6240-6245$.

[13] Yasui K, Konishi C, Gen Y, Endo M, Dohi O, Tomie A, et al. EVI1, a target gene for amplification at 3q26, antagonizes transforming growth factor- $\beta$-mediated growth inhibition in hepatocellular carcinoma. Cancer Sci. 2015; 106: 929-937.

[14] Brooks DJ, Woodward S, Thompson FH, Dos Santos B, Russell M, Yang JM, et al. Expression of the zinc finger gene EVI-1 in ovarian and other cancers. Br J Cancer. 1996; 74: $1518-1525$.

[15] Queisser A, Hagedorn S, Wang H, Schaefer T, Konantz M, Alavi S, et al. Ecotropic viral integration site 1, a novel oncogene in prostate cancer. Oncogene. 2017; 36: 1573-1584.

[16] Tanaka M, Ishikawa S, Ushiku T, Morikawa $T$, Isagawa $T$, Yamagishi M, et al. EVI1 modulates oncogenic role of GPC1 in pancreatic carcinogenesis. Oncotarget. 2017; 8: 99552 99566.

[17] Evan GI, Vousden KH. Proliferation, cell cycle and apoptosis in cancer. Nature. 2001; 411: 342-348.

[18] Baldazzi C, Luatti S, Zuffa E, Papayannidis C, Ottaviani E, Marzocchi G, et al. Complex chromosomal rearrangements leading to MECOM overexpression are recurrent in myeloid malignancies with various $3 \mathrm{q}$ abnormalities. Genes Chromosomes Cancer. 2016; 55: 375-388.

[19] Zhu H, Gan X, Jiang X, Diao S, Wu H, Hu J. ALKBH5 inhibited autophagy of epithelial ovarian cancer through miR7 and BCL-2. J Exp Clin Cancer Res. 2019; 38: 163.

[20] Zhang X-M, Liu Z-L, Qiu B, Xu Y-F, Pan C, Zhang Z-L. Downregulation of EVI1 expression inhibits cell proliferation and induces apoptosis in hilar cholangiocarcinoma via the PTEN/AKT signalling pathway. J Cancer. 2020; 11: 14121423.

[21] Wu L, Wang T, He D, Li X, Jiang Y. EVI-1 acts as an oncogene and positively regulates calreticulin in breast cancer. Mol Med Rep. 2018; 19: 1645-1653.

[22] Wieser R. The oncogene and developmental regulator EVI1: expression, biochemical properties, and biological functions. Gene. 2007; 396: 346-357.

[23] Konantz M, André MC, Ebinger M, Grauer M, Wang H, Grzywna S, et al. EVI-1 modulates leukemogenic potential and apoptosis sensitivity in human acute lymphoblastic leukemia. Leukemia. 2013; 27: 56-65.

[24] Wang H, Schaefer T, Konantz M, Braun M, Varga Z, Paczulla $\mathrm{AM}$, et al. Prominent oncogenic roles of EVI1 in breast carcinoma. Cancer Res. 2017; 77: 2148-2160.

[25] Lu Y, Liang Y, Zheng X, Deng X, Huang W, Zhang G. EVI1 promotes epithelial-to-mesenchymal transition, cancer stem cell features and chemo-/radioresistance in nasopharyngeal carcinoma. J Exp Clin Cancer Res. 2019; 38: 82. 